\section{Multi- objective robust design optimization of an engine mounting system}

\author{
E. Courteille and F. Mortier \\ C.F. GOMMA Barre Thomas S.A.
}

L. Leotoing and E. Ragneau
INSA Rennes (LGCGM)

\begin{abstract}
This paper introduces a new method to support designers to find optimal and robust solutions of engine mounting system. The mounting system design is a compromise between isolation of the vehicle from engine vibration and constraining the motion of the powertrain within vehicle packaging. Based on the classical pendulum mounting system of a front wheel drive vehicle with a transversely four-cylinder engine, this study deals with the definition of a new global engine mounting concept for the NVH (Noise Vibration and Harshness) improvement of the vehicle characteristics at idle speed. The practical application of the numerical optimization is complicated by the fact that engine mounting system is a stochastic system. Its characteristics have a probabilistic nature. Multi-Objective Genetic Algorithm (MOGA), i.e. Pareto-optimization, is taken as the appropriate framework for the definition and the solution of the addressed multi-objective robust optimization problem. An experimental correlation analysis has been conducted on a Pareto-optimal solution to show the model accuracy.
\end{abstract}

\section{INTRODUCTION}

In vehicles, the engine mounts play an essential role for the NVH comfort. The main functions of these mounts (rubber or hydraulic) are to provide static supports for the engine and to isolate the vibrations of the engine from the rest of the vehicle. Thus, the design of this vehicle part, becoming of a great complexity, constitutes an essential step for the NVH improvement. Idle shake is an important factor in determining the low-frequency vehicle comfort. Among this, the Torque Roll Axis (TRA) decoupling method is frequently a starting point in the industrial mounting system design practice [1]. This method concentrates on minimizing the powertrain roll vibration for only crankshaft pulsating torque at idle speed, without considering the unbalance force due to the up-down motion of the pistons applied at the engine center. The background theory of this technique is widely described in literature without clearly analyzing the NVH improvement of the dynamic chassis responses
$[2,3,4]$. We extend the TRA decoupling method with two objectives which improve directly through the design of the engine mounting system the vehicle responses.

Optimization of the powertrain system modal alignment and packaging constraints are performed on an analytical model consisting of rigid body representations of the engine and vehicle with non-linear engine mounts. In literature, many efforts were devoted to the development of more accurate modeling strategies than the synthesis model which is presented here. Some studies include driveline, exhaust system, and Finite Element model of the chassis [5, 6]. As a good definition of the rest of the vehicle is still not available in early stages of the vehicle design process, we preferred to describe the design parameters by a probability distribution. Random deviations of the system's parameters lead to a random change in system's efficiency. The designer must consider the robustness of the solution, which defines the system response to be insensitive to the variation of the input parameters working effectively in a range of circumstances. Traditional optimization techniques tend to "overoptimize", producing solutions that perform well at the design point but have poor off-design characteristics. Multi-Objective Genetic Algorithms (MOGA), i.e. Paretooptimization, are used to set the framework of the multiobjective robust design optimization study of the engine mounting system. A MATLAB / FRONTIER procedure for the preliminary design of the engine suspension systems has been developed, relying on the described method.

An experimental correlation analysis has been conducted on a test bench to show the accuracy of the vehicle model for a quantification of vehicle comfort when idling. The predicted and measured full vehicle responses are compared on a robust Pareto-optimal solution defined by the optimization procedure.

\section{DESIGN CONSIDERATIONS IN ENGINE MOUNTING}

Once the vehicle level NVH targets at passenger interface points (driver's ear sound, seat track, etc) have been determined by a benchmarking analysis, a multibody simulation can be used very efficiently to optimize the location, number, and properties of engine mounts in an earlier design stage.

\section{MULTI-BODY SIMULATION MODEL}

In derivation of the equations of motion to simulate static and dynamic behaviors of engine mount systems with supporting structures, a good modeling of a total vehicle system can consist in four subsystems : powertrain, engine mounts, chassis and suspensions. Since small displacements can be assumed, powertrain is modeled as a rigid body of time-invariant inertial matrix of dimension 6 . The powertrain is supported by an arbitrary 
number of mounts to the vehicle chassis, also modeled as a rigid body elastically suspended. The connection to the ground is simply represented by four systems of linear spring and viscous damper in parallel at each wheel, characterized by their stiffness and damping coefficients following the three directions of the vehicle frame coordinates. The previous assumptions have become standard practice for the development of system simulation models of vehicles.

In the automotive industry the rigid body motions are commonly referred to bounce (vertical), lateral, and longitudinal for the translation modes and roll, pitch, and yaw for the rotational modes. For the powertrain, roll is the rotation about the $Y$ axis of the vehicle frame (Figure 1 ), pitch about the $X$ axis and yaw the rotation about the $Z$ axis. Conversely, the vehicle chassis roll is its rotation about the $\mathrm{X}$-axis (longitudinal) and pitch the rotation about the $\mathrm{Y}$-axis (lateral).

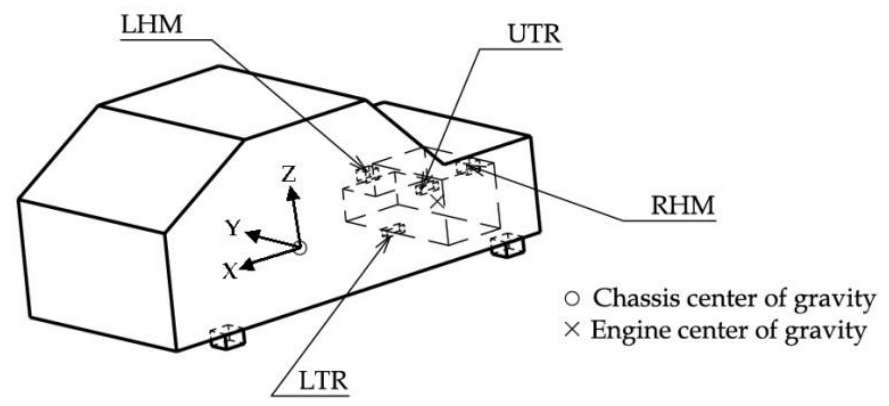

Figure 1 : Simulation model of a vehicle.

The current mounting system for 4 cylinder front wheel drive cars is typically made up of three mounts, two of which bear most of the weight of the powertrain while the last is a roll-restrictor. The two mounts bearing the static weight are located on the body rails. These are referred as Right Hand Mount (RHM) and Left Hand Mount (LHM) (Figure 1 and 2). The roll-restrictor attaches the lower part of the engine to the rear cross member of the front subframe. It is referred as the Lower Torque Rod (LTR). A second roll-restrictor is used on the upper part of the engine for high torque engine. It is referred as the Upper Torque Rod (UTR).

\section{ENGINE MOUNTING CONCEPTS}

The first step in developing the engine mounting system is to design for the static requirements of the powertrain. For different gears corresponding to driving conditions of the vehicle, the engine is loaded with a given percentage of the maximum available torque which is considered constant in time (static analysis). It provides the operating points of the mounts on their forcedisplacement characteristics. The stiffnesses are consequently linearized by the value of the tangent at the operating point. Therefore, by using these stiffnesses, the model enable to assess the rigid body modes of the powertrain in the vehicle as well as to analyze the motion of the powertrain and the chassis under various engine operating conditions (idle, full load sweep) and road/wheel inputs.

\section{POWERPLANT INTEGRATION ISOLATION STRATEGIES}

\section{ENGINE EXCITATION}

In this study, we focus on the NVH improvement of a front wheel drive vehicle with diesel four-cylinder engine without balance-shaft. The theoretical knowledge of the engine excitation is essential to explain the vibration level of idle shake [7], and to choose best engine mounting design, considering the $\mathrm{NVH}$ target required (general layout, engine mount specifications).

The engine excitation force can be divided into two sources. The first of these is the unbalance force due to the up-down motion of the piston, and the second is the torque variance due to the pressure change in the cylinder during the explosion cycle. To reach satisfactory levels of comfort, it is necessary to consider all the transfer paths, in all the directions.

The dominant excitation of four-cylinder engines for front wheel drive vehicles at low speed range is the secondorder torque fluctuation caused by change in the gas pressure inside the cylinder. At high speed, the secondorder unbalance force is dominant. Torque due to gas pressure and torque due to inertia force are in opposition of phase. For a certain speed, the torque due to inertia force has the same value as the torque due to gas pressure, the acyclism is minimized then as well as the powertrain roll vibrations (between 2500 and 3500 rpm).

\section{TORQUE ROLL AXIS DECOUPLING CONCEPT}

The Torque Roll Axis (TRA) decoupling method is commonly used to reduce the engine roll excitation at the mounts in the case of a transverse pendulum mounting system [1]. The TRA is defined as the resulting fixed axis of rotation of the unconstrained three dimensional powertrain (i.e. either free or supported elastically on very soft springs) when the pulsating torque is applied along the crankshaft [2, 3]. The TRA method consists in conditioning the on-ground powertrain to oscillate about the TRA with the crankshaft torque variation excitation in all frequency range. The background theory of this technique is widely described in literature without clearly analyzing the $\mathrm{NVH}$ improvement of the dynamic chassis responses.

The interactions with the chassis should be evaluate to determine the significance of the powertrain rigid body modes decoupling condition for on-ground system to its in-vehicle NVH behavior. In fact, the couplings with the chassis can strongly modify the powertrain vibratory response and then cancel out the TRA decoupling condition. The TRA decoupling condition is still effective in the full vehicle model only if the interactions between the engine and the chassis are low, i.e. at frequencies where the coupling order between the powertrain and 
the chassis is low, beyond the eigenvalues of the rigid body modes [8]. This configuration is respected for a front wheel drive vehicle with diesel four-cylinder engine. The fundamental frequency of the idle excitation for a four-cylinder engine (second-order at $26.6 \mathrm{~Hz}$ for 800 $\mathrm{rpm}$ ) is in the range of isolation, beyond the frequencies of the rigid body modes (classically between 4 to $18 \mathrm{~Hz}$ ).

The practical interest of the TRA decoupling scheme is that there is only one pure mode excited and then only one frequency to move away from the excitation frequency to reduce powertrain roll vibration. Using a pendulum mounting layout increases the degrees of freedom in tuning, since the longitudinal stiffness characteristics of the mounts do not affect in the powertrain vertical motion. It means that the TRA decoupled mode frequency is quite easy to decrease while respecting the static requirements to bear the powertrain weight. The engine mounts must present in the longitudinal direction a good compromise frequency filtration / static requirements under full engine load conditions in the lowest gears (first and second gear). Accordingly, the mounts should operate in a linear stiffness region in the longitudinal direction to still satisfy the TRA decoupling condition under normal operating condition. This will help minimize structureborne noise transmission into the vehicle for the entire engine speed range. The mounts must be designed to increase their longitudinal stiffness only during maximum static torque condition in the lowest gears [2, 3].

\section{COUNTERBALANCEMENT OF VEHICLE MOTION}

A four-cylinder engine strongly vibrates, as a whole, in rolling, but also in vertical. The resultant of unbalance forces due to the up-down motion of the pistons is applied at the engine center. The center of gravity of the powertrain, provided with a gearbox, is shifted compared to the center of the engine (distance $d$ on figure 2). The motion of the powertrain is not any more a simple vertical translation but a combination of translation and pitching, which, in general, tends to decrease the gearbox side vertical vibrations. All the points are not equivalent. The position of nodes of vibration are sensitive and one can use them to establish the engine mounting layout.

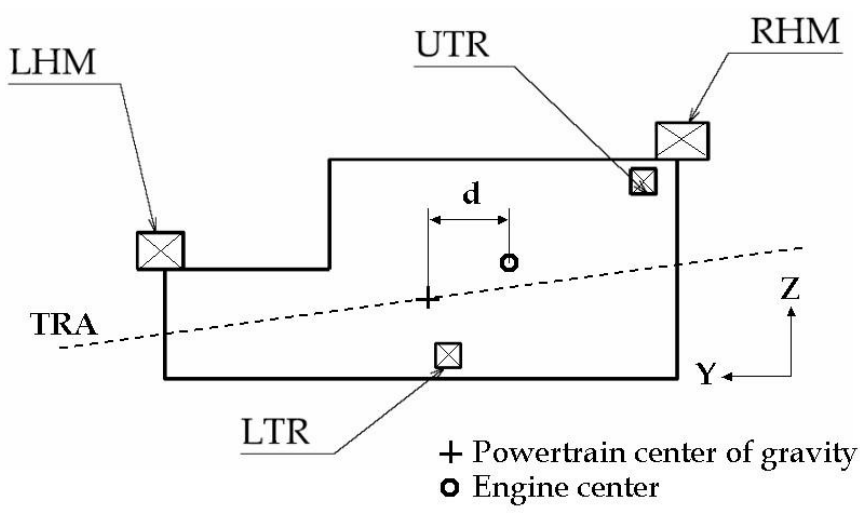

Figure 2 : Engine mounting system.

In a completely modal decoupled on-ground powertrain, all the rigid body modes are independent of each other, i.e. an excitation input produces a response only in that direction. Conversely, a powertrain coupled for pitch and bounce modes, would behave such that a vertical input produces both a vertical response as well as pitch response. A torque pitch input produces a pitch response as well as vertical. Decoupling should be related to a specific excitation.

Once the TRA decoupling axiom is satisfied, the overall level of vertical vibration of the vehicle chassis is dominated by the force transmitted through the vertical stiffness of LHM and RHM related to the control of powertrain bounce and pitch motions. To improve idle vibration, it is possible to reduce the dynamic spring rate of these dominant paths for the sake of lower input force transmitted, or to use the phase of the input force into counterbalancing the sum of the resulted vehicle motion contributions. The first solution presents limitation because of the vertical spring rate of LHM and RHM are directly related to the powertrain static bearing. A minimum stiffness level is required.

Depending on the coupling level of powertrain pitchbounce modes, the unbalance forces due to the updown motion of the pistons applied to the engine center could produce a vertical input force of LHM in phase opposition with the vertical input force of RHM. In such a configuration, these vertical input forces brought back to the center of gravity of the vehicle chassis would produce vertical and pitch as well as roll movements. By the way of rigid body motion phases, the rolling of the vehicle chassis either counterbalances the vertical and pitch contributions on the left car side or increases them on the right car side. It is interesting in so far as the vertical acceleration response at the driver's seat floor (LHSF) for a left drive car should be cancelled out by a perfect balance of movement contributions for one engine speed. Such a configuration is graphically explained on an engine mounting design obtained by the following optimization procedure.

\section{ENGINE SUSPENSION OPTIMISATION : PROBLEM FORMULATION}




\section{DESIGN VARIABLES}

One method widely used by the automotive community to represent the force-displacement characteristics of a rubber component is the non-linear spline curve approach. It assumes that the deformations in the three local coordinate directions of the mount are independent to one another such that three non-linear spline curves can be defined in these directions. The relationship between the force component $\left(F_{j}\right)$ and the relative displacement $\left(\Delta \mathrm{l}_{\mathrm{j}}\right)$ along each axis (j) of the mount reference frame, can be well approximated by means of an order $n(n=3,5$ or 7 ; figure 3$)$. This is a correct assumption that allows a substantial reduction of the number of parameters that have to be tuned. Three design variables summarize the nonlinear behavior :

- $\mathrm{K}_{\mathrm{j} 0 \text { stat }}[\mathrm{N} / \mathrm{mm}]$ the spring stiffness,

- $\Delta \mathrm{l}_{\mathrm{jmax}}[\mathrm{mm}]$ the maximum value of relative displacement under the known force $F_{j \max }$,

- $\mathrm{n}[-]$ the exponent of the non-linear characteristic of the mount.

The elastic force is given by :

$$
\mathrm{F}_{\mathrm{j}}=\mathrm{K}_{\mathrm{j} 0 \text { stat }} \cdot \Delta \mathrm{l}_{\mathrm{j}}+\left(\left(\mathrm{F}_{\mathrm{j} \max }-\mathrm{K}_{\mathrm{j} 0 \text { stat }} \cdot \Delta \mathrm{l}_{\mathrm{j} \max }\right) / \Delta \mathrm{l}_{\mathrm{j} \max }\right) \cdot \Delta \mathrm{l}_{\mathrm{j}}^{\mathrm{n}}
$$

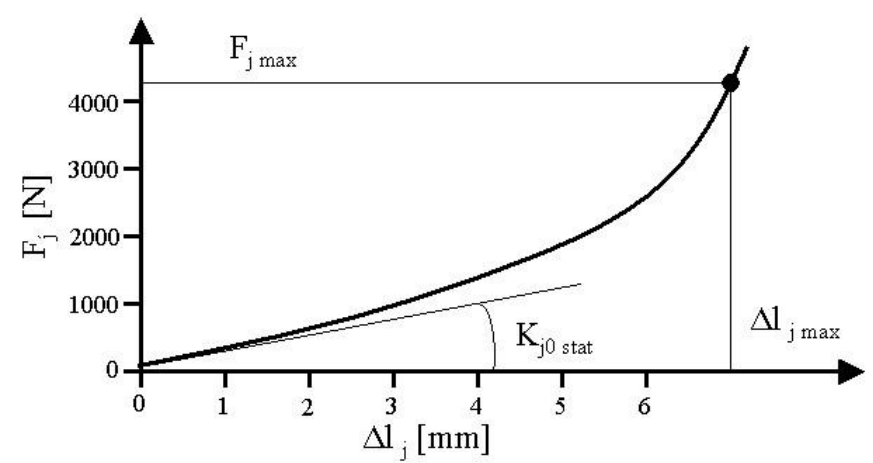

Figure 3 : force_displacement curve for a rubber mount.

The working point of the mount is given by the displacement and rotation of the engine under the action of its weight and the torque in the running load condition. The value of the stiffness $\mathrm{K}_{\text {jstat }}$ used in the dynamic computations is obtained by the tangent to the characteristic curve at the working point :

$$
\mathrm{K}_{\mathrm{j} \text { stat }}=\left.\frac{\mathrm{dF}_{\mathrm{j}}}{\mathrm{d} \Delta \mathrm{l}_{\mathrm{j}}}\right|_{\text {working-point }}
$$

The stiffness of natural rubber is increasing lightly with frequency. This is called "dynamic stiffening". The phase is quite independent of frequency. Dynamic stiffening is strongly correlated with the phase angle, which is itself correlated with the filler content. The structural damping of the rubber can be well approximated by using an equivalent viscous damping model. Accordingly, it is convenient to define the dynamic stiffness $\overline{\mathrm{K}_{\mathrm{j}}}$ as :

$\overline{\mathrm{K}_{\mathrm{j}}}=\beta \mathrm{K}_{\mathrm{jstat}}+\mathrm{j} \omega \mathrm{C}_{\mathrm{j}}$ with $\mathrm{C}_{\mathrm{j}}=\frac{\beta \mathrm{K}_{\mathrm{jstat}} \tan \left(\phi_{\mathrm{j}}\right)}{\omega}$

$\beta$ is an appropriate coefficient accounting for the dynamic stiffening of the rubber at low frequencies (e.g., at about $25 \mathrm{~Hz}, \beta$ can range from 1.1 to 1.8). The phase angle $\phi_{j}$ for the rubber element is quite constant with frequency (4 to 8 degrees), so that the equivalent viscous damping $C_{j}$ increases with linear stiffness, while decreases with frequency.

Finally, by using the above mentioned formulation, the maximum number of independent parameters needed to completely define the force-displacement curves of a single rubber mount along all directions is 6 , once $\Delta \mathrm{l}_{\mathrm{jmax}}$ and the order $\mathrm{n}$ are defined. The dynamic factor and the damping parameters are also fixed. A torque rod has a stiffness characteristic only along the axial direction. 3 additional design variables are necessary to define each mount location.

With reference to the suspension layout under investigation, 30 design variables are tuned, which refer to the position and the stiffness characteristics in each direction of the powertrain mounts (LHM and RHM), and the lateral position and the axial stiffness of the torquerods (LTR and UTR).

\section{PERFORMANCE INDEXES}

The statement of the optimisation problem is defined according to the previous section.

\section{TRA decoupling index}

A complete dynamic TRA decoupling mode depends on whether a rigid body mode of the on-ground powertrain can be forced to be in the TRA direction. Starting with the undamped equations of motion for the on-ground powertrain, the condition for a complete TRA decoupling mode is defined by the following relationship :

$$
\left[\mathbf{K}_{\text {engine }}\right]\left\{\mathrm{q}_{\text {TRA }}\right\}=\omega_{\text {TRA }}^{2}\left[\mathbf{M}_{\text {engine }}\right]\left\{\mathrm{q}_{\text {TRA }}\right\}
$$

where :

- $\left\lfloor\mathbf{M}_{\text {engine }}\right\rfloor$ is the generalized square mass matrix of dimension 6 ,

- $\left\lfloor\mathbf{K}_{\text {engine }}\right\rfloor$ is the generalized square stiffness matrix of dimension 6 , 
- $\left\{\mathrm{q}_{\mathrm{TRA}}\right\}$ is the TRA direction (vector of dimension 6),

- $\omega_{\text {TRA }}$ is the frequency of the TRA decoupling mode.

Through this expression, the frequency of the TRA decoupling mode is simultaneously set to $10.5 \mathrm{~Hz}$, then minimizing the powertrain roll vibration coming from the crankshaft pulsating torque at idle speed.

The TRA decoupling index is defined as :

TRA $=\left\|\left[\mathbf{K}_{\text {engine }}\right]\left\{\mathrm{q}_{\text {TRA }}\right\}-\omega_{\text {TRA }}^{2}\left[\mathbf{M}_{\text {engine }}\right]\left\{\mathrm{q}_{\text {TRA }}\right\}\right\|_{2}$

where $\|\cdot\|_{2}$ is the 2-norm.

$\underline{\text { Response at the driver's seat floor }}$

We carry out study to reduce the vertical acceleration response at the driver's seat floor through a counterbalance of the rolling of the vehicle chassis with the pitch and bounce movements coming from the resultant of unbalance forces due to the up-down motion of the pistons. The performance index is the value of the driver's seat floor vertical acceleration at idle speed (26.6 Hz at $800 \mathrm{rpm})$, assuming that a perfect balance of movement contributions is produced when it is minimized. The accelerations are expressed in $\mathrm{m} / \mathrm{s}^{2}$. To facilitate reading of the acceleration levels, a logarithmic ordered scale is used with a reference equal to $1 \mathrm{~m} / \mathrm{s}^{2}$.

\section{$\underline{\text { Kinetic energy of the chassis }}$}

The TRA decoupling index and the vertical acceleration response of the driver's seat floor at the idle speed $(800$ rpm) but also the global kinetic energy of the vehicle chassis are simultaneously minimized at the idle range between 20 to $40 \mathrm{~Hz}$ for the vehicle system. The total kinetic energy transmitted by the engine to the vehicle chassis can be written as:

$\kappa_{\text {chassis }}=\frac{1}{2} \omega^{2}\left\{\mathrm{q}_{\text {chassis }}\right\}^{\mathrm{T}}\left[\mathbf{M}_{\text {chassis }}\right]\left\{\mathrm{q}_{\text {chassis }}\right\}$

where $\left[\mathbf{M}_{\text {chassis }}\right]$ is the generalized square mass matrix and $\left\{q_{\text {chassis }}\right\}$ is the generalized displacement of the vehicle chassis. The Root Mean Square (RMS) value of the global kinetic energy of the vehicle chassis in the idle range is used as target $\mathrm{NVH}$ index.

This criterion makes it possible to minimize globally the vehicle vibrations by a distribution of the kinetic energy transmitted by the engine mounting system to the weakest inertia terms of the chassis.

\section{Constraints}

In addition to these main objectives, a number of packaging constraints have to be set. The engine mounts displacements and the engine rotations must not exceed proper threshold values during different static manoeuvres (full engine load in lowest gears, braking, cornering, etc). Furthermore, the powertrain bounce mode is sought between 8 and $10 \mathrm{~Hz}$ to avoid interaction with the front vehicle suspensions modes for road/wheel inputs.

\section{SEARCH METHOD}

\section{PARETO-OPTIMAL SOLUTIONS}

Solving this optimization problem with multiple conflicting objectives across a high-dimensional design space is a difficult goal. While in single-objective optimization the optimal solution is usually clearly defined, this does not hold for multi-objective optimization problems. Instead of a single optimum, there is rather a set of alternative trade-offs, generally known as Pareto-optimal solutions. These solutions are optimal in the wider sense that no other solutions in the search space are superior to them when all objectives are considered. Given a multiobjective minimization problem defined by $k$ objective functions, a solution vector $x_{i}$ is Pareto-optimal (i.e. nondominated) if a solution vector $x_{j}$ doesn't exist such that (figure 4) :

$$
\begin{aligned}
& \forall \mathrm{n} \in\{1,2, \ldots \mathrm{k}\}: \mathrm{f}_{\mathrm{n}}\left(\mathrm{x}_{\mathrm{j}}\right) \leq \mathrm{f}_{\mathrm{n}}\left(\mathrm{x}_{\mathrm{i}}\right) \\
& \quad \text { and } \exists \mathrm{m} \in\{1,2, \ldots, \mathrm{k}\}: \mathrm{f}_{\mathrm{m}}\left(\mathrm{x}_{\mathrm{j}}\right)<\mathrm{f}_{\mathrm{m}}\left(\mathrm{x}_{\mathrm{i}}\right)
\end{aligned}
$$

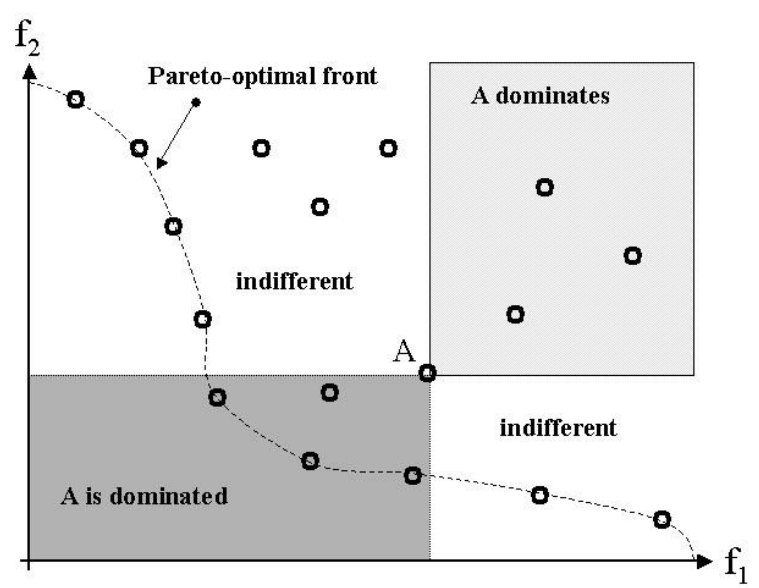

Figure 4 : Illustrative example of Pareto optimality in the 2-objective space.

Evolutionary algorithms possess several characteristics that are adequate for this problem and make them preferable to classical optimization methods. Various evolutionary approaches to multi-objective optimization have been proposed since 1985, capable of searching for multiple Pareto-Optimal solutions concurrently in a single simulation run [9]. The commercial optimization program FRONTIER and the technical computing 
software MATLAB are used to set the framework of the multi-objective robust design optimization study of the engine mounting system. The Multi-objective Genetic Algorithm (MOGA), implemented by Fonseca and Fleming [10], is used to perform the optimization problem.

\section{ROBUST DESIGN}

Unlike the deterministic optimization problem, the robust design optimization considers the probabilistic functional of the objective functions. The designer has to take into account the robustness of the solution, which defines the system response to be insensitive to the variation of the input parameters working effectively in a range of circumstances. Various factors of uncertainty can thus be present in the geometry of the system or material properties of the mount due to manufacturing variability.

The generalized treatment of such problems is to use probabilistic or stochastic models instead of the deterministic model inside the optimization loop. The deterministic model is replaced by an iterative stochastic model in an uncertainty space. The uncertainty space is represented in terms "mean" (MEAN) and "standard deviation" (STDV) of the input and output variables. Using FRONTIER's MORDO (Multi-Objective Robust Design Optimization ) approach, it's possible to look for the best robust design. For these reasons, MORDO evaluates two different values for each objective :

- The average value of the performance index inside the variables distribution;

- The standard deviation that should be minimized or at least keep under control (i.e. constrained).

\begin{tabular}{|c|c|}
\hline & Standard deviation \\
\hline $\mathrm{K}_{\mathrm{j} 0 \text { stat }}[\mathrm{N} / \mathrm{mm}]$ & $5 \%$ \\
\hline$\Delta \mathrm{l}_{\mathrm{j} \max }[\mathrm{mm}]$ & $5 \%$ \\
\hline $\mathrm{X}$ coordinate $[\mathrm{mm}]$ & $2 \mathrm{~mm}$ \\
\hline $\mathrm{Y}$ coordinate $[\mathrm{mm}]$ & $2 \mathrm{~mm}$ \\
\hline $\mathrm{Z}$ coordinate $[\mathrm{mm}]$ & $5 \mathrm{~mm}$ \\
\hline
\end{tabular}

Table 1 : Standard deviation of the design variables.

A normal distribution with a standard deviation is imposed on all the design variables of the engine mounting system (Table 1). For each input variable value, $\mathrm{N}$ sample designs are created according to a normal distribution (centered in that point) and with the fixed perturbation nearby the point. To simulate the scatter in the values for the location of the centers of gravity, masses, and mass-moments of inertia of the powertrain and vehicle body, we fix virtually an additional point-mass to each rigid body. Their locations are randomly chosen in a cube around the nominal location of the center of gravity (500 $\mathrm{mm}$ side), and the additional mass is defined by a normal distribution with a zero mean and $5 \%$ of the nominal mass as standard deviation.

In order to control the perturbation of the responses of the vehicle chassis in the uncertainty space, it's mandatory to add constraint or objective on performance indexes, in addition to the objectives on the mean (Table 2).

\begin{tabular}{|l|c|l|}
\hline \multirow{2}{*}{ TRA decoupling index. } & \multirow{2}{*}{ TRA } & minimize MEAN \\
\cline { 3 - 3 } & & minimize STDV \\
\hline $\begin{array}{l}\text { RMS value of the kinetic } \\
\text { energy of the vehicle } \\
\text { chassis in the idle range } \\
\text { (20 to } 40 \mathrm{~Hz}) .\end{array}$ & $\mathrm{K}$ & minimize MEAN \\
\cline { 3 - 3 } & & minimize STDV \\
\hline $\begin{array}{l}\text { Value of the driver's seat } \\
\text { floor vertical acceleration } \\
\text { at idle speed (800 rpm). }\end{array}$ & LHSF_Z & minimize MEAN \\
\cline { 3 - 3 } & & minimize STDV \\
\hline
\end{tabular}

Table 2 : Summary of the defined performance indexes.

In almost all robust optimization problems, evaluating uncertain functions is computationally very intensive. The accuracy of the estimates for the mean and the standard deviation is particularly important. However, this accuracy is dependent on the number of the sample and, obviously, the number required for a given accuracy depends on several factors such as the type of uncertainty and the number of variables.

\section{GLOBAL OPTIMIZATION PROCESS}

The algorithm will attempt a number of evaluation equal to the size of the initial population for the MOGA algorithm multiplied by the number of sample and the number of generation. A rule of thumb would suggest possibly to accumulate an initial population possibly more than $2^{*}$ number_of_variables*number_of_objectives. Thus, the initial population is generated by a random sequence of 360 designs (30 design variables, 6 objectives ; table 2). The constraints handling method is based on penalty functions. The concept is that the fitness function decreases according to the intensity of the constraint violation. 
The major disadvantage of the MOGA technique is mainly related to the number of evaluations necessary to obtain satisfactory solutions. The search for the optimal solutions extends in all the directions from design space and produces a rich data base and there is not a true stop criterion. The uniformity and the richness of the data base are very useful for the capitalization and the statistical analysis of the results. In the context of engine mounting design, the numerical evaluation of the performances calls upon MATLAB codes is not expensive in terms of computing time (about $2 \mathrm{~s}$ ). The size of the run is usually defined by the computing resources available. In an attempt to solve the optimization problem in an acceptable timeframe, the number of the sample was fixed to 20 and the number of generations evaluated is 10 . The system will always check if one design evaluation has been already performed and will eventually skip the computation. The required computation time for the global optimization process is about 40 hours (1.7 GHz / 1 Gb RAM).

To reduce this computing time, still too significant in an industrial context, ones can use approximations of the functions objectives and constraints. The Response Surface Methodology (RSM) makes it possible to have an approximation of the functions objectives in near instantaneous time. A study in this field is underway.

\section{ENGINE SUSPENSION OPTIMIZATION : NUMERICAL RESULTS}

The figure 5 and 6 highlight the MOGA algorithm convergence toward TRA decoupling and the vertical acceleration at the driver's seat floor. The points represented with a triangle are unfeasible designs since they do not respect the constraints.

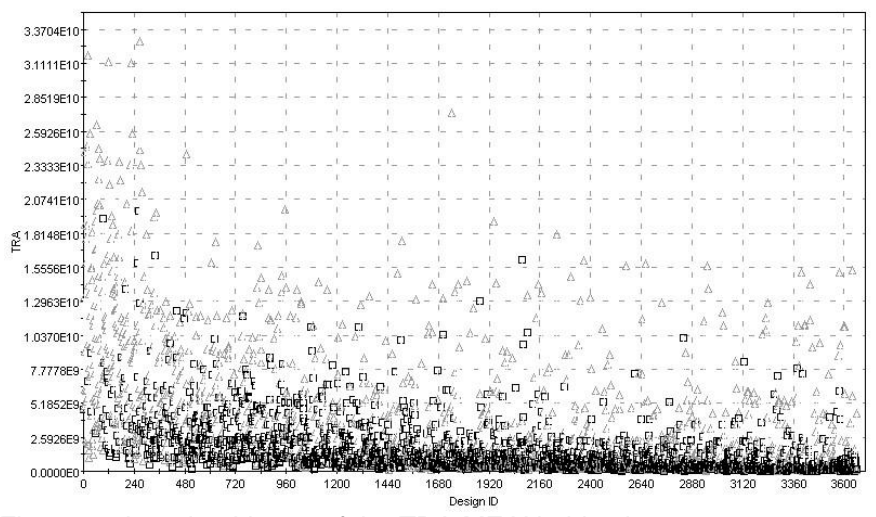

Figure 5 : Iteration history of the TRA:MEAN objective.

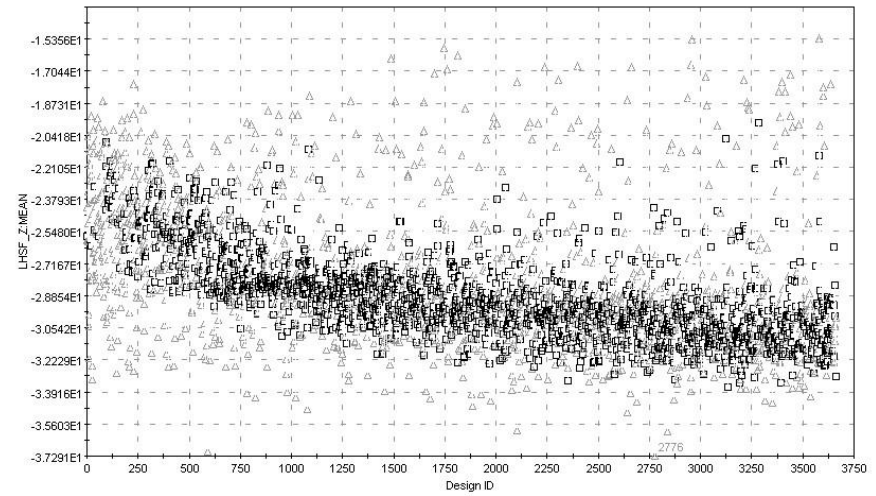

Figure 6 : Iteration history of the LHSF Z:MEAN objective (in dB, reference $\left.1 \mathrm{~m} / \mathrm{s}^{2}\right)$.

Figure 7 shows the projection of the resulting Paretooptimal sets onto the LHSF_Z:MEAN / LHSF_Z:STDV domain, stressing the sensible improvement that can be obtained for the two objectives respecting the constraints. The right-down region of the plot is principally characterized by a non-feasibility against the constraint on the powertrain bounce mode frequency. This constraint is the most penalizing.

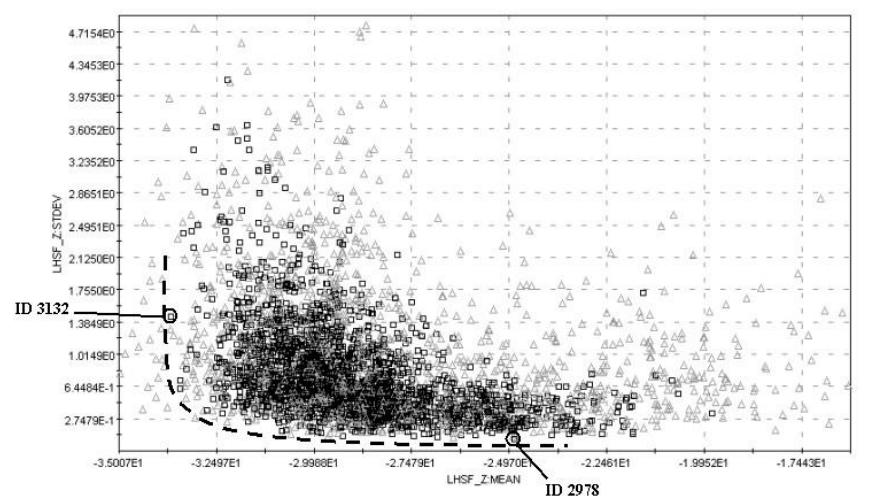

Figure 7 : Scatter chart of LHSF_Z:MEAN versus LHSF_Z:STDV

The most interesting characteristic of figure 7 is the shape of the Pareto-front on these two objectives. The Pareto-front is very wide (dashed line on figure 7). This aspect indicates that there is a conflict between the two objectives. The best solution from the vertical driver's seat floor acceleration viewpoint is the design ID 3132. This solution performs well at the design point but have poor off-design characteristics, i.e. the system response is sensitive to the variation of the input parameters $($ STDV $=1.4 \mathrm{~dB})$. In such a configuration, the vertical acceleration response at the driver's seat floor is reduced through a counterbalance of the rolling of the vehicle chassis with the pitch and bounce movements coming from the resultant of unbalance forces due to the up-down motion of the pistons (Figure 8). 


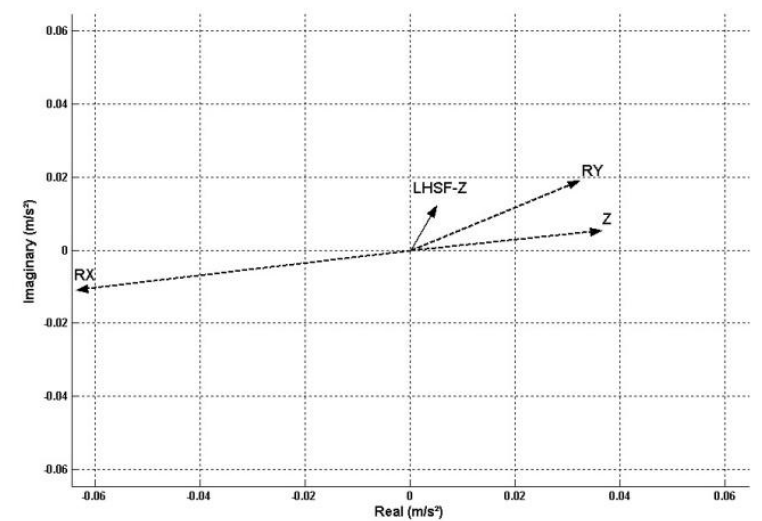

Figure 8 : Contribution of each vehicle chassis movement for LHSF_Z (ID 3132).

On account of the packaging constraints and a participation of the powertrain roll vibration on the pitch movement of the vehicle chassis, even low with a TRA mode at $10.5 \mathrm{~Hz}$, the counterbalanced cannot be completely fulfilled. The minimization of the acceleration is limited to the idle speed $(26.6 \mathrm{~Hz}$ at $800 \mathrm{rpm})$ because the unbalance forces of inertia increase with the engine speed (Figure 9). The response is reduced by an adequate repartition of the RHM and LHM vertical forces through the geometrical and inertial properties of the vehicle chassis for only one engine speed. A little variation on the input parameters will perturb the counterbalance.

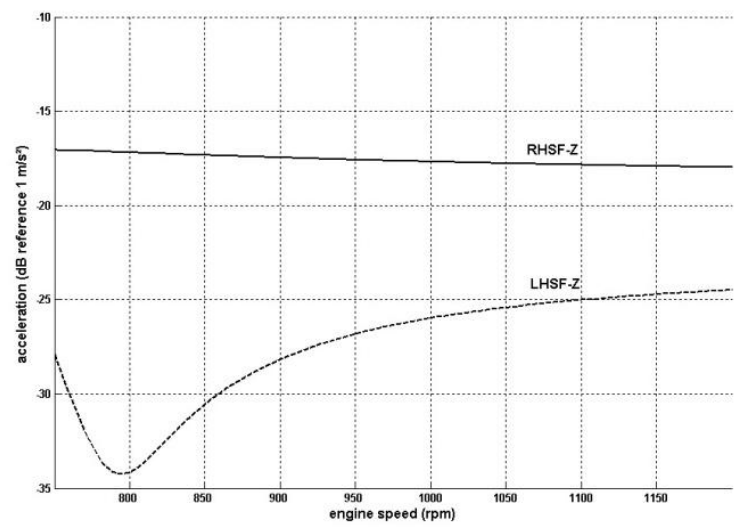

Figure 9 : Vertical acceleration at the driver's seat floor (LHSF_Z) and at the passenger's seat floor (RHSF_Z) (ID 3132).

As it can be noticed from the plot of figure 10, the minimization of the vertical acceleration at the driver's seat floor increases the global level of the kinetic energy of the vehicle chassis. The excitation of the roll mode of the vehicle chassis penalizes the right car side (Figure 11). It is obvious that the final solution is a compromise among different and conflicting objectives. The richness of the solutions available enables the designer to choose the best compromise among the optimal solutions while being conscious of the working specifications. It is important to understand that the final selection remains always subjective and a decision of the designer.

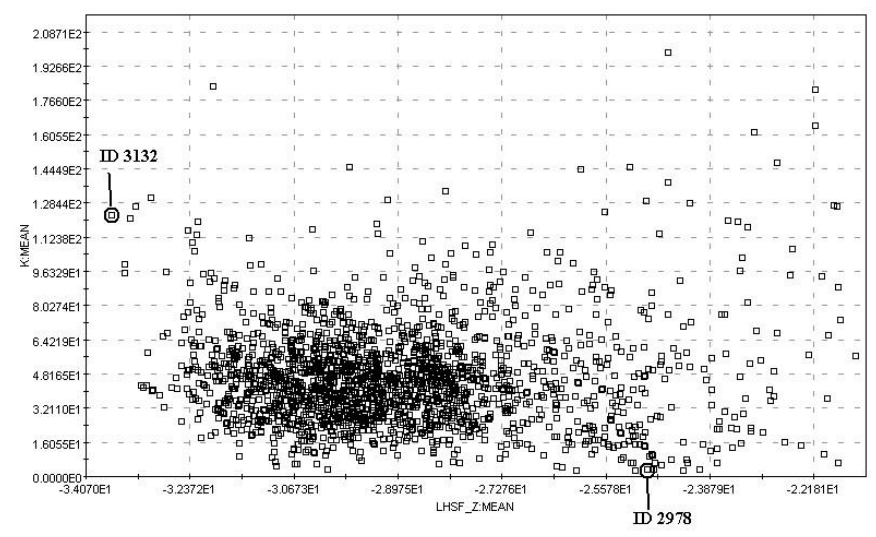

Figure 10 : Scatter chart of LHSF_Z:MEAN versus K:MEAN with only feasible designs.

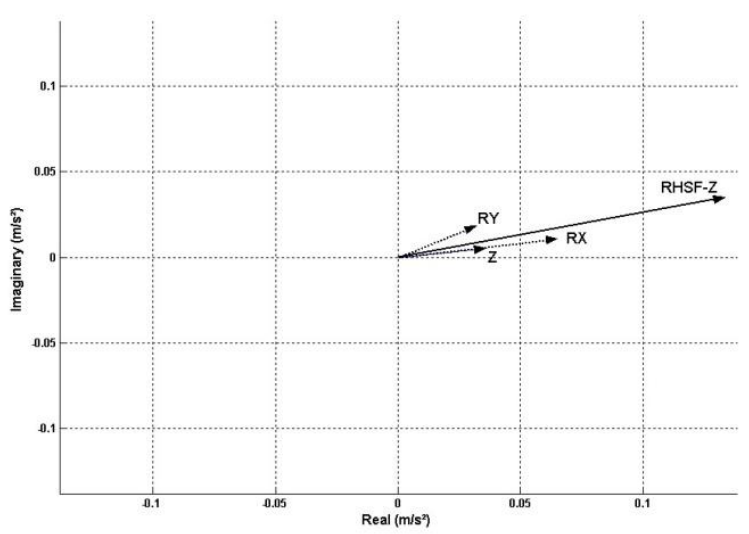

Figure 11 : Contribution of each vehicle chassis movement for the passenger's seat floor vertical acceleration (RHSF_Z) (ID 3132).

\section{EXPERIMENTAL VALIDATION}

The accuracy of the vehicle model is validated by the comparison of the predicted and measured full vehicle responses. RENAULT engineers has developed a test bench that can be used for a quantification of vehicle comfort when idling as well as for road excitation testing. The validation of the model has been conducted on a engine mounting design which minimizes the global level of the kinetic energy of the vehicle chassis (Figures 7 and 10 : ID 2978). This solution presents a good robustness at simulation toward the chassis accelerations at the target points (LHSF and RHSF). Three types of measurements are performed for comparison with the simulation, i.e. :

- Triaxial acceleration at the powertrain-side and body-side of the mounts,

- Input force transmitted trough each mount along the longitudinal axis,

- Vertical and longitudinal acceleration measurements at the passenger and driver seat floor locations.

The theoretical calculation for powertrain accelerations at mount attachment shows fairly good correlation in its amplitude and phase with the measured $2^{\text {nd }}$ order idle 
accelerations (Figure 12). The acceleration levels on the powertrain-side of the mounts are very sensitive to the mass-moment of inertia of the powertrain, which is an input data quite difficult to assess experimentally.

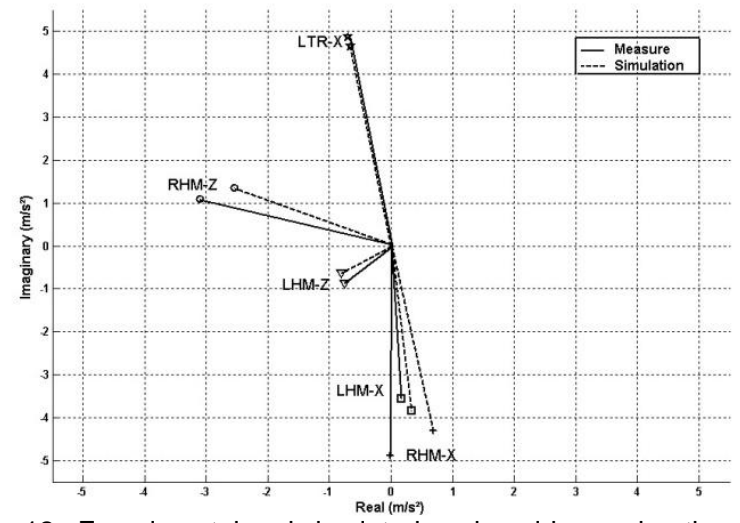

Figure 12 : Experimental and simulated engine-side acceleration of the mounts at idle speed (second-order at $26.6 \mathrm{~Hz}$ for $800 \mathrm{rpm}$ ).

The vibration path is tightly related to the dynamic stiffness of the mounts. In order to evaluate the dynamic spring rate of each engine mount during the test conditions, input forces were measured using force transducers in the longitudinal direction. The dynamic stiffness of the mount is the ratio between applied force and displacement obtained by the frequency spectra of the acceleration signal measured at the points before and after the mount. The dynamic spring rate of each engine mount was also determined in lab test. As it can be noticed in table 3, the dynamic characteristics in operating conditions can be significantly different from the a priori corresponding lab measurement. This discrepancy is a result of inaccurate preload and excitation amplitude applied to the mount during the lab test.

\begin{tabular}{|c|c|c|}
\hline & Test lab & $\begin{array}{l}\text { Operating } \\
\text { conditions }\end{array}$ \\
\hline KX_LHM & $150 \mathrm{~N} / \mathrm{mm}$ & $105 \mathrm{~N} / \mathrm{mm}$ \\
\hline KX_RHM & $72 \mathrm{~N} / \mathrm{mm}$ & $79 \mathrm{~N} / \mathrm{mm}$ \\
\hline KX_UTR & $290 \mathrm{~N} / \mathrm{mm}$ & $290 \mathrm{~N} / \mathrm{mm}$ \\
\hline KX_LTR & $320 \mathrm{~N} / \mathrm{mm}$ & $310 \mathrm{~N} / \mathrm{mm}$ \\
\hline
\end{tabular}

Table 3 : Longitudinal dynamic stiffness of the mount.

As figures 13 and 14 show, the model could be regarded as an accurate model only if the different factors of uncertainty present in the material properties of the mount or in the geometry of the system are taken into account. The measured accelerations at the target points matched well the uncertainty calculated domain created by 100 sample designs centered in the nominal design. There is an important correlation between the left and right longitudinal acceleration as shown in figure 12. Once the TRA decoupling axiom is satisfied, only the pitch movement of the vehicle chassis generates longitudinal acceleration, which is almost symmetric for the left and right side of the car.

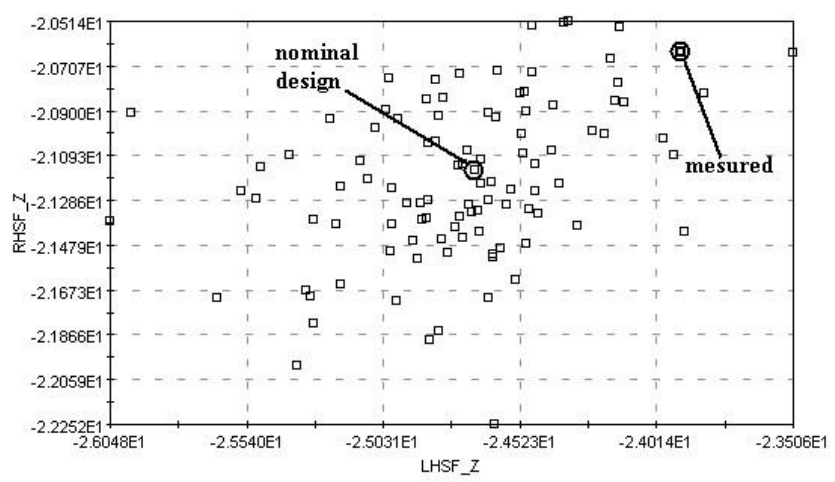

Figure 13 : Scatter chart of LHSF Z versus RHSF Z in the robust design space around the nominal design ID 2978 for 100 sample designs.

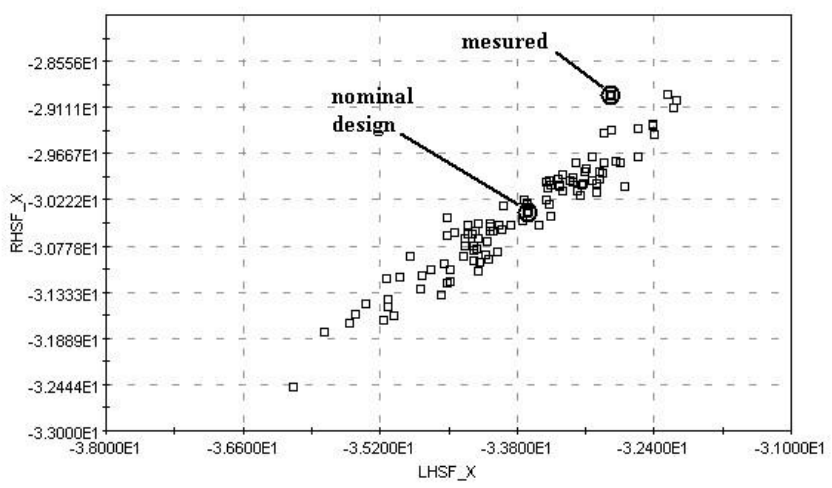

Figure 14 : Scatter chart of LHSF_X versus RHSF_X in the robust design space around the nominal design ID 2978 for 100 sample designs.

\section{CONCLUSION}

Design and optimization must be done in a short period of time and, as a result, an automated MATLAB / FRONTIER design procedure for finding an optimum engine mounting system is developed. The presented optimization, even if subjected to further refinements (Response Surface Methodology), fulfills the industrial demands in the preliminary design of the engine suspension systems : optimize simultaneously comfort and packaging requirements in an acceptable timeframe while considering the robustness of the solution.

The interactive use of evolutionary multi-objective algorithm in the engine mounting design is very attractive from the engineering viewpoint. Paretooptimization may be considered as a tool providing a set of efficient solutions among different and conflicting objectives, under different constraints. Furthermore, the designer has to take into account the robustness of the solution. Accordingly, it is possible that the best solution is not the same of the best stable solution. The final 
choice remains always subjective and is left to the designer.

Quick and satisfactory results can be obtained in the simulation of idle shake through a good selection of parameters without using a complex model. But it is planed to improve model preciseness by performing test and analysis simultaneously in other operating conditions (key on / key off, engine bounce, etc).

\section{ACKNOWLEDGMENTS}

This work was partly developed for a mechanical engineering degree thesis, in collaboration with RENAULT. The cooperation of RENAULT engineers is gratefully acknowledged. The authors also acknowledge ESTECO and SIREHNA for providing a FRONTIER software license.

\section{REFERENCES}

1. R.M Brach, "Automotive Powerplant Isolation Strategies", SAE Paper 971942, 1994.

2. P.E. Geck and R.D. Patton, "Front wheel drive engine mount optimization", SAE Paper 840739, 1984.

3. R. Singh and T. Jeong, "Analytical Methods of Decoupling the Automotive Engine Torque Roll
Axis", Journal Sound and Vibration 234, pp. 85-114, 2000.

4. A. Khajepour and A. Geisberger, "Engine Mounting Systems from Passive to Active", Recent Research Development Sound and Vibration, 91-7895-031-6 pp. 697-718, 2002.

5. J.H. Kim, J.M. Lee and H.J. Yim, "Dynamics and Design Optimization of Elastically Engine-Mount Systems", SAE Paper 945086, 1994.

6. M. Sirafi and M. Qatu, "Accurate Modeling for the Powertrain and Subframe Modes", SAE Paper 200301-1469, 2003.

7. B. Swoboda, "Mécanique des moteurs alternatifs", Ecole Nationale Supérieure du Pétrole et des Moteurs, 1984.

8. E.Courteille, F. Mortier, L. Leotoing and E. Ragneau, "Analytical study of coupling between subsystems of a vehicle NVH model", International Conference on Noise and Vibration Engineering ISMA, 2004.

9. G.B. Lamont and D.A. Van Veldhuizen, "Multiobjective Evolutionnary Algorithms : Analyzing the State-of-the-Art", Massachusetts Institute of Technology, 2000.

10. C.M. Fonseca and P.J. Fleming, "Multiobjective Optimization and Multiple Contraint Handling with Evolutinary Algorithms", IEEE Transactions on Systems, Man, and Cybernetics, 28 pp 26-47, 1998. 